\title{
Abrupt Severe Chest Pain and Vomiting: Remember to Think of a Ruptured Oesophagus (Boerhaave Syndrome)
}

\author{
Deeba Ali ${ }^{1}$, Arnaud Detroz $^{1}$, Yilmaz Gorur ${ }^{2}$, Lionel Bosquée ${ }^{3}$, Benoît Cardos ${ }^{4}$, Carla Cobanoiu ${ }^{5}$, Noel Lorenzo Villalba ${ }^{6}$ \\ ${ }^{1}$ Service des Urgences, CHU Sart Tilman, Liège, Belgium \\ ${ }^{2}$ Service de Radiologie, CHU Sart Tilman, Liège, Belgium \\ ${ }^{3}$ Service de Pneumologie, Clinique André Renard, Herstal, Belgium \\ ${ }^{4}$ Département des Sciences de la Santé Publique, Université de Liège, Liège, Belgium \\ ${ }^{5}$ Service de Neurologie, CHC Saint-joseph, Liège, Belgium \\ ${ }^{6}$ Service de Médecine Interne, Diabète et Maladies Métaboliques, Hôpitaux Universitaires de Strasbourg, Strasbourg, France
}

\section{Doi: 10.12890/2019_001265-European Journal of Case Reports in Internal Medicine - @ EFIM 2019}

Received: $27 / 08 / 2019$

Accepted: $17 / 09 / 2019$

Published: 04/10/2019

How to cite this article: Ali D, Detroz A, Gorur Y, Bosquée L. Cardos B. Cobanoiu C. Villalba NL. Abrupt severe chest pain and vomiting: remember to think of a ruptured oesophagus (boerhaave syndrome). EJCRIM 2019;6: doi:10.12890/2019_001265.

Conflicts of Interests: The Authors declare that there are no competing interest

This article is licensed under a Commons Attribution Non-Commercial 4.0 License

\section{ABSTRACT}

Boerhaave syndrome or spontaneous rupture of the oesophagus is a severe condition commonly misdiagnosed or unrecognized. Prognosis is poor even if the diagnosis is made promptly. We describe a case of Boerhaave syndrome diagnosed after the development of pneumomediastinum and cardiac arrest. Unfortunately, the patient died 48 hours after admission to the Intensive Care Unit. This entity requires a multidisciplinary management approach which may include conservative, surgical or endoscopic procedures.

\section{LEARNING POINTS}

- Boerhaave syndrome is a diagnostic and therapeutic challenge, and rapid diagnosis and management are crucial.

- A thoraco-abdominal CT scan with oesophageal opacification is the gold standard investigation.

- A multidisciplinary and individualized approach is needed in the management of this condition.

\section{KEYWORDS}

Boerhaave syndrome, pneumomediastinum, conservative, surgical, endoscopic

\section{INTRODUCTION}

Spontaneous perforation of the oesophagus, also referred to as Boerhaave syndrome, is the longitudinal rupture of a previously unremarkable oesophagus ${ }^{[1]}$. This spontaneous perforation commonly results from the sudden increase of pressure within the oesophageal lumen caused by uncoordinated oesophageal motility during forceful vomiting. The diagnosis is difficult due to the incidental nature of the disorder and because its symptoms are often inconsistent and non-specific. Boerhaave syndrome must be treated as a life-threatening emergency as it has a mortality rate of up to $40 \%[2]$.

\section{CASE DESCRIPTION}

A 90-year-old patient with a history of hypertension, diabetes type 2 and chronic alcoholism was admitted to the emergency department for cough, dyspnoea and abdominal pain. The initial work-up was consistent with pulmonary and urinary tract infections and so empiric treatment with ceftriaxone ( $1 \mathrm{~g}$ daily) was initiated. 
On day 2 of admission in the geriatric department, the patient abruptly presented sharp chest pain accompanied by severe shortness of breath and vomiting. On physical examination, he was tachycardic (heart rate 100 beats/min), blood pressure was $100 / 60 \mathrm{mmHg}$, respiratory rate was 30 per min and oxygen saturation on room air was $85 \%$. He was afebrile and saturation increased to $95 \%$ under 3 litres of oxygen. Diffuse rales were heard in both pulmonary fields. No murmurs or pericardial rubbing was noted. The abdomen was not painful with any masses. Subcutaneous crepitus in the chest was noted.

Blood tests showed mild hyperleukocytosis $\left(14,000 \mathrm{~mm}^{3}\right)$, elevated C-reactive protein (80 mg/l) and hyponatraemia (128 mmol/l). Liver, renal and coagulation tests were within normal ranges. Arterial blood gas analysis revealed hypoxaemia $\left(\mathrm{pO}_{2}: 60 \mathrm{mmHg}\right)$ and $\mathrm{metabolic}$ acidosis. The electrocardiogram was normal. A chest x-ray showed left paramediastinal clarity (pneumomediastinum), and cervical and axillary subcutaneous emphysema without associated pneumothorax.

The patient was transferred to the ICU. A few hours after admission, he experienced severe sinus bradycardia (33 beats/min), hypotension and stupor which preceded cardiac arrest. The patient was resuscitated.

A second chest $x$-ray showed the previously described lesions were stable. Once he was haemodynamically stable, the patient was sent for a thoracic scan which showed a left pleural effusion and a prominent pneumomediastinum with suspicion of a distal oesophageal breach.

These results were consistent with Boerhaave syndrome (Figs. 1-3). Broad-spectrum intravenous antibiotic therapy with meropenem was initiated. However, surgical treatment was not undertaken in light of the patient's comorbidities and general condition. Endoscopic treatment was proposed but refused by the family. Supportive treatment was started and the patient died 48 hours later.

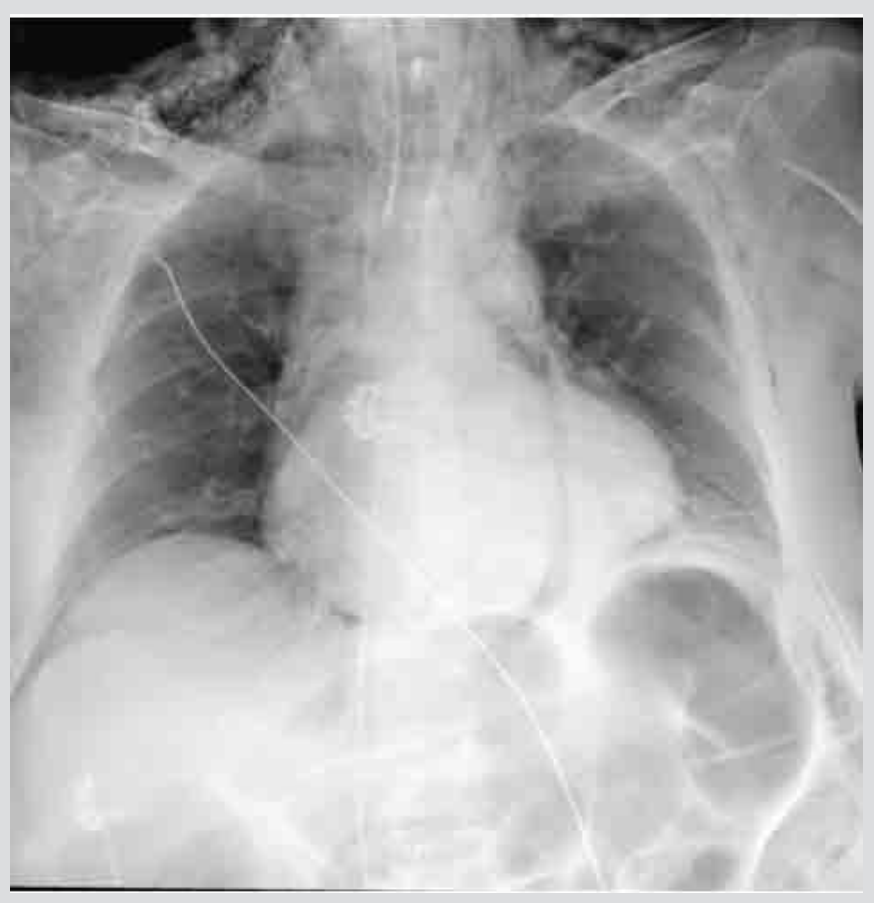

Figure 1. Chest radiograph showing a significant pneumomediastinum with subcutaneous emphysema in the cervical and axillary regions. A left pleural effusion is also seen

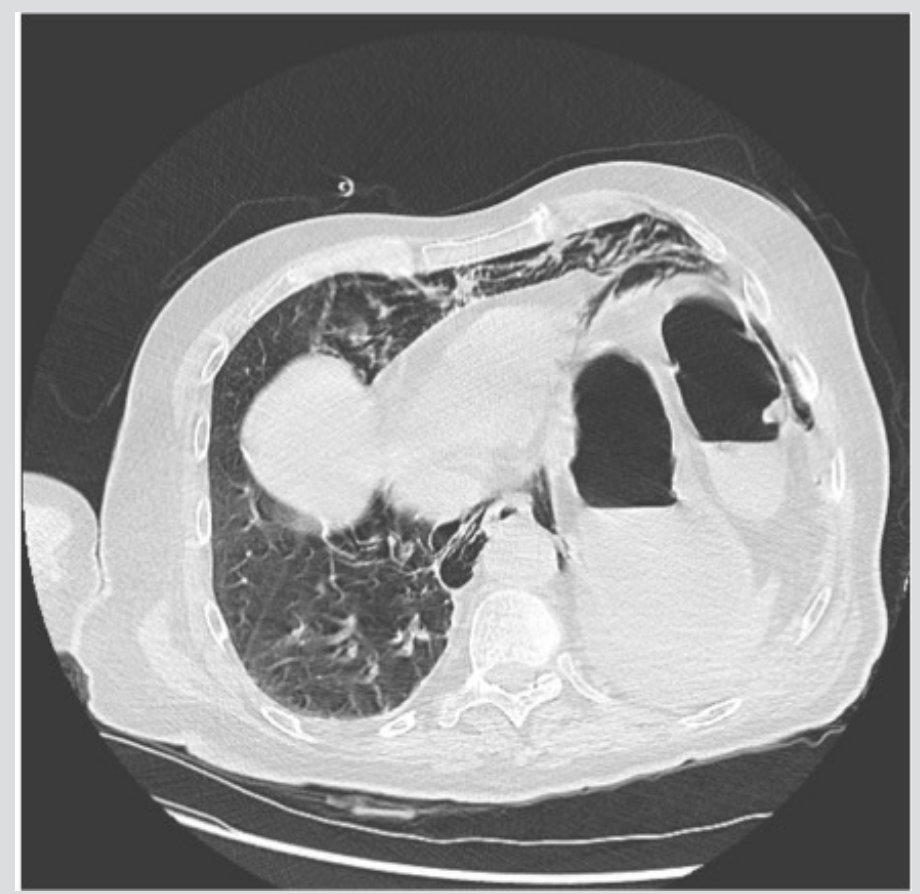

Figure 2. Non-enhanced CT scan showing a large pneumomediastinum surrounding the oesophagus. A small effusion is seen in the left pleura. No pulmonary mass, pneumothorax or bone lesion is seen in the thorax

\section{DISCUSSION}

Boerhaave syndrome or spontaneous rupture of the oesophagus is a rare and often fatal condition. It is defined as complete rupture of the oesophageal wall following a sudden increase in intra-oesophageal pressure as the result of intraluminal hyperpressure with a closed glottis. It can also result from faecal vomiting, an epileptic seizure, an asthma attack or the Heimlich manoeuvre ${ }^{[1]}$. This entity has been mainly described in men ( $80 \%$ of cases) between the fourth and sixth decades of life. 


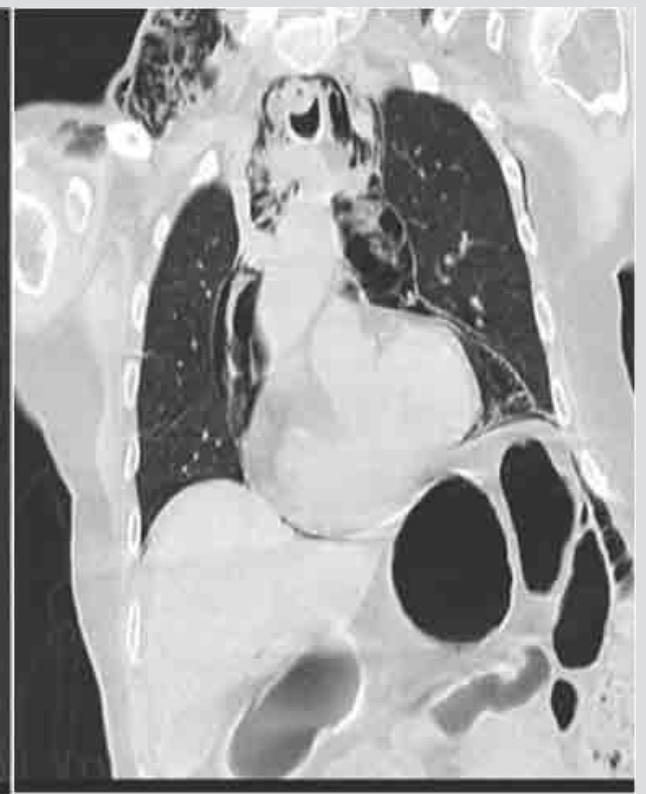

Figure 3. Non-enhanced CT scan showing a large pneumomediastinum surrounding the oesophagus. A small effusion is seen in the left pleura. No pulmonary mass, pneumothorax or bone lesion is seen in the thorax

The mortality rate varies from $28 \%$ to $85 \%$ depending on how quickly treatment is initiated ${ }^{[1,2]}$. Rupture is more common in the lower third and on the left side of the oesophagus, due to parietal sheet weakness. The clinical picture is non-specific, making diagnosis difficult. The chest pain and dyspnoea do not help diagnosis as they are frequently observed in other diseases. There can be signs of sepsis and shock as a result of mediastinal, pleural or abdominal infection.

The presence of vomiting, chest pain and subcutaneous emphysema is known as Mackler's triad, which is pathognomonic but rarely seen in clinical practice. Laboratory investigations are not helpful as they are non-specific. Chest x-ray may show indirect and non-specific signs such as pneumomediastinum, pneumoperitoneum, subcutaneous emphysema and unilateral or bilateral pleural effusion.

A thoracic CT scan is the gold standard for diagnosis and has a sensitivity of $92-100 \%$. It usually shows pneumomediastinum and allows identification of the oesophageal breach by contrast leak ${ }^{[2,3]}$. Performance of an upper gastrointestinal endoscopy is controversial despite its excellent sensitivity (100\%) and specificity (92\%) because it may increase injuries and favour mediastinal contamination.

In this case, diagnosis was based upon the anamnesis, the clinical picture and the results of the chest x-ray and scan which allowed tension pneumothorax and tension pneumomediastinum to be ruled out.

The management of Boerhaave syndrome includes medical, endoscopic or surgical procedures, depending on clinical stability. Medical treatment consists of early broad-spectrum empiric antibiotics, intravenous proton pump inhibitors and parenteral nutrition. Surgery is required if clinical worsening occurs during medical management,. Patients with underlying comorbidities who are unlikely to tolerate surgery may benefit from endoscopic therapy with a self-expandable stent. There is no real consensus about management since no prospective randomized trials have yet been published ${ }^{[4,5]}$. In this case, surgical management was not an option given the comorbidities of the patient and endoscopic treatment was refused by the family.

Increasing life expectancy means patients require a case-by-case approach, where the risks and benefits of invasive treatments for each patient are carefully considered.

\section{CONCLUSION}

Boerhaave syndrome is a rare condition difficult to diagnose. It is a serious medical emergency, as in this case. Prognosis is poor and survival depends on early diagnosis and treatment. Management must be multidisciplinary and individualized. 


\section{REFERENCES}

1. Rokicki M, Rokicki W, Moj M, Bsoul T, Rydel M. Boerhaave syndrome - over 290 years of surgical experiences. Can the disorder recur? Pol Przegl Chir 2018;91(3):27-29.

2. Swol J, Ficker JH, Mannke B. Cervical emphysema in Boerhaave syndrome. Dtsch Arztebl Int 2019;116(12):211.

3. Le Cam Y, Tuel N, Bellier JD. Rupture spontanée de l'œsophage: il faut y penser devant toute douleur thoracique. Réan Urg 1998;7:415-417.

4. Dayen C, Mishellany H, Hellmuth D, Mayeux I, Aubry P, Glerant JC, et al. La rupture spontanée de l'œsophage ou syndrome de Boerhaave: à propos de trois cas et revue de la littérature. Rev Mal Resp 2001;18(5):537-540.

5. Lu H, Carron PN, Godat S, Pittet R. Syndrome de Boerhaave: mise au point sur la physiopathologie, le diagnostic et le traitement précoce. Rev Med Suisse 2018;14:299-303. 